\section{Molecular network design}

SIR - Naturally occurring materials have a positive Poisson's ratio $^{1}$; when stretched, the material becomes thinner. Recently, synthetic materials have been processed that exhibit a negative Poisson's ratio, (becoming fatter when stretched $)^{2-6}$. Such materials can have improved mechanical properties, such as enhanced shear moduli, indentation resistance and fracture toughness ${ }^{2,7}$. All these materials exhibit a negative Poisson's ratio as a result of microstructures, or geometric units, that are at least tens of micrometres in size. We have used molecular modelling techniques to design a molecular network with a negative Poisson's ratio. By altering the geometry of the repeat unit, Poisson's ratio can be varied to produce positive or negative values with mechanical properties that are either isotropic or anisotropic.

The Poisson's ratio of a material is given by $v_{x y}=-\varepsilon_{y} / \varepsilon_{x}$, where $\varepsilon_{x}$ is an applied tensile strain and $\varepsilon_{y}$ is the resulting tensile strain in the transverse direction. For isotropic materials $v_{x y}=v_{y x} \equiv v$ with the limits $-1<v<1 / 2$. For anisotropic materials neither of these limits apply. For example, orthotropic materials may have Poisson's ratios anywhere within the range $\left|v_{x y}\right|<\left(E_{x} / E_{y}\right)^{1 / 2}$, where $E_{x}$ and $E_{y}$ are Young's moduli in the orthogonal directions $x$ and $y^{8,9}$.

Examples of materials that have been fabricated with a negative Poisson's ratio
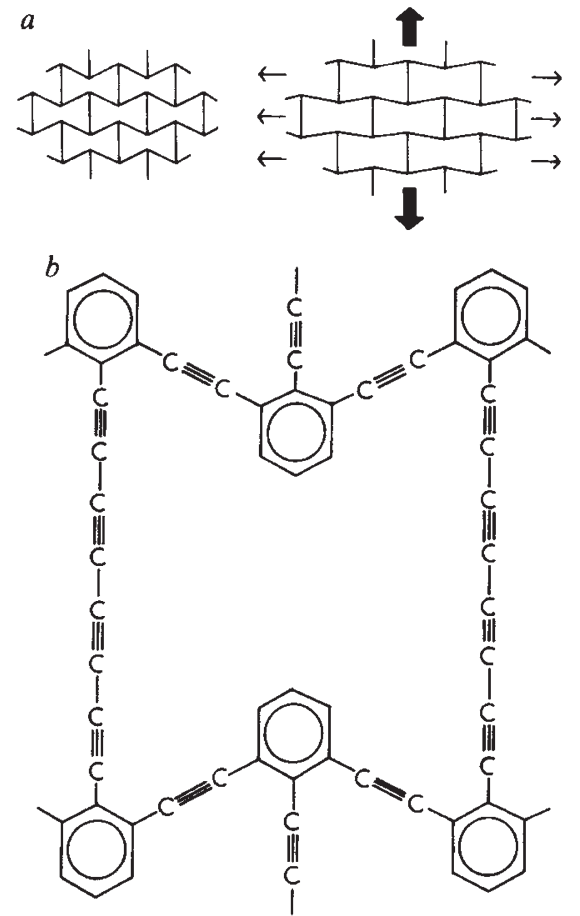

a, Two-dimensional re-entrant honeycomb, showing transverse expansion on stretching. $b$, $(1,4)$-reflexyne with a negative Poisson's ratio.

are two-dimensional honeycombs ${ }^{4}$, three-dimensional foams ${ }^{2,3}$ and microporous polymers $5,6,10$. An example of the simplest microstructure that produces

general the simple flexure model overestimates Poisson's ratio relative to the molecular-mechanics approach. This is because both stretching and rotational this effect is illustrated sche-

matically in Fig. $a$. A con- CALCULATED VALUES OF POISSON'S RATIOSFOR $(n, m)$-REFLEXYNE ventional hexagonal honeycomb has a positive Poisson's ratio, but making the cells 're-entrant', as in the figure, produces a negative Poisson's ratio.

The honeycombs and foams manufactured so far have low densities and are intrinsically weak and flexi-

ble. By reproducing this geometric unit on the molecular scale, however, it should be possible to take advantage of the innate free volume found, for example, in polymeric structures to increase the density and absolute stiffness of the material. Figure $b$ illustrates a molecular network structure that reproduces, on the molecular scale, the features of the geometry in Fig. $a$. We suggest nonsystematic naming convention for the hexagonal network of the form $(n, m)$ flexyne for the honeycomb structure, where $n$ is the number of acetylene links on the diagonal branches and $m$ the number of links on the vertical branches, and $(n, m)$-reflexyne for the re-entrant structure. By altering the values of $n$ and $m$, the structure can be either isotropic or anisotropic with varying Poisson's ratios. The use of benzene rings at the junctions and acetylene groups along the branches creates, in principle, a planar two-dimensional structure. Although such a network may be hard to synthesize, it provides a simple example for molecular tailoring of Poisson's ratio.

A model has been developed ${ }^{11}$ that successfully predicts Poisson's ratio for macroscopic honeycombs. This model assumes that deformation occurs by flexure, with no stretching of the cell arms, whereas in the molecular structure of Fig. $b$ deformation will occur by flexing, stretching and rotation of the arms. We have used a molecularmechanics program ${ }^{12}$ which incorporates a standard valence force field to model the deformation of this structure and thus to calculate Poisson's ratio. Because of the symmetry of the network, only one unit cell need be modelled to determine the network's deformation behaviour. A force is applied in the $x$ direction and the resulting displacements in the $x$ and $y$ directions are used to obtain $v_{x y}$. Similarly, a force in the $y$ direction is used to obtain $v_{y x}$. Strains of less than $5 \%$ are used to ensure elastic behaviour.

In the table we give values for five $(n, m)$-reflexyne networks. The reentrant structure does indeed produce negative Poisson's ratios, although in
Flexure model

Molecular mechanics

longitudinal deformation at the expense of the transverse. The value of Poisson's ratio can be altered by changing the shape of the repeat unit. $v_{x y}=1$ represents a truly isotropic structure. $v_{x y}=$ -1 is only quasi-isotropic in that Poisson's ratios measured by stretching along directions away from the principal axes will have different values; it is a squaresymmetric structure.

The molecular network described here represents a first attempt at designing a material that demonstrates a negative Poisson's ratio owing to mechanisms acting at the molecular level. We are now investigating more complex structures that are more amenable to synthesis that have been proposed by $\mathrm{G}$. Wei and S. F. Edwards of Cambridge University. To avoid the cumbersome phrase 'negative-Poisson's-ratio materials', we suggest that they be called auxetic materials, or auxetics (from the Greek auxetos: that may be increased, referring to the width and volume increase when stretched).

K.E. EVANS M. A. NKANSAH

Department of Materials Science and Engineering,

University of Liverpool, PO Box 147 , Liverpool L69 3BX, UK

I. J. HUTCHINSON S. C. ROGERS

Research and Technology Group,

ICl Chemicals and Polymers Ltd,

PO Box 8, The Heath,

Runcorn WA7 4QE, UK

1. Landau, L. D. \& Lifshitz, E. M. Theory of Elasticity (2nd edn), 10-15 (Pergamon, Oxford, 1970)

2. Lakes, R. S., Science 235, $1038-1040$ (1987).

3. Friis, E. A., Lakes, R. S. \& Park, J. B. J. Mat. Sci. 23, 4406-4414 (1988)

4. Gibson, L. J. \& Ashby, M. F. Cellular Solids: Structure and Properties 70-82 (Pergamon, Oxford, 1988)

5. Caddock, B. D. \& Evans, K. E. J. Phys. D. 22 1877-1882 (1989)

6. Evans, K. E. \& Caddock, B. D. J. Phys. D 22, 1883 1887 (1989).

7. Evans, K. E. Chem. Ind. 20, 654-657 (1990).

8. Evans, K. E. J. Phys. D22, 1870-1876 (1989)

9. Lempriere, B. M. Am. Inst. Aeronaut. Astronaut. J. 6 2226-2227 (1968)

10. Alderson, K. L. \& Evans, K. E. Polymer (submitted).

11. Gibson, L. J. Ashby, M. F., Schajer, G. S. \& Robertson C. I. Proc. R. Soc. Lond. A382 25-42 (1982).

2. DISCOVER, Biosym Technologies Inc., 10065 Barnes Canyon Road, San Diego, Catifornia 92121, USA 